\title{
Pattern formation from consistent dynamical closures of uniaxial
}

\section{nematic liquid crystals}

\author{
Humberto Híjar* and Diego Marquina de Hoyos \\ Facultad de Ciencias, Universidad Nacional Autónoma de México, \\ Circuito Exterior de Ciudad Universitaria, 04510, D. F., México \\ Iván Santamaría-Holek ${ }^{\dagger}$ \\ UMJ-Facultad de Ciencias, Universidad Nacional Autónoma \\ de México Campus Juriquilla, Querétaro 76230, México
}

* Fellow of SNI Mexico. E-mail for correspondence: hijar@daad-alumni.de

$\dagger$ Fellow of SNI Mexico. E-mail for correspondence: isholek.fc@gmail.com 


\begin{abstract}
Pattern formation in uniaxial polymeric liquid crystals is studied for different dynamic closure approximations. Using the principles of mesoscopic non-equilibrium thermodynamics in a mean-field approach, we derive a Fokker-Planck equation for the single-particle non-homogeneous distribution function of particle orientations and the evolution equations for the second and fourth order orientational tensor parameters. Afterwards, two dynamic closure approximations are discussed, one of them considering the relaxation of the fourth order orientational parameter and leading to a novel expression for the free-energy like function in terms of the scalar order parameter. Considering the evolution equation of the density of the system and values of the interaction parameter for which isotropic and nematic phases coexist, our analysis predicts that patterns and traveling waves can be produced in lyotropic uniaxial nematics even in the absence of external driving.
\end{abstract}

PACS numbers:

Keywords: Patterns, Uniaxial liquid crystals, Isotropic-Nematic transition, Doi-Hess theory 


\section{INTRODUCTION}

The study of anisotropic fluids is a very active field of research in soft condensed matter $\underline{\underline{1}} \underline{\underline{19}}$ in which important results have been achieved very recently. $\frac{18}{19}$ One of the main aspects of this study is the obtention of free energies able to describe the transition from orientational disordered to ordered states in relation to density or temperature conditions. $\underline{1}, \underline{2}$ The evaluation of this free-energy is a difficult $\operatorname{task}^{\underline{7}}$ in which mean field theories of anisotropic molecules in suspensions or pure systems have proven to be very powerful. The two general approaches to the determination of the free-energy function can be categorized as equilibrium and dynamic ones, and lead to different nontrivial structural and dynamic properties of nematic liquid crystals. $\underline{1}, \underline{2}, \underline{4}, \underline{7}, 18,19$

Equilibrium approaches are focused on the direct calculation of the canonical distribution function, the partition function and the free-energy by taking into account the general symmetry properties of the system. $11,14,19,20$ For uniaxial systems, this implies that the fourth order scalar orientational parameter can be expressed in terms of the second order scalar

orientational parameter $\stackrel{1}{*}$ Recently, a step forward in the equilibrium approach has been given in Ref. [19] where, by using the maximum entropy principle based on Gibbs entropy postulate, $\stackrel{11}{\Perp}$ a very general method for calculating the free-energy was proposed. In contrast, dynamic approaches to the problem are based on a closure of the evolution equations for the hierarchy of moments of the non-equilibrium distribution function, typically obtained from a Fokker-Planck equation. $1,2,7,18,21$ An excellent analysis of the physical consistency of several dynamic closures proposed in the literature is given in Ref. [18].

We consider that these two approaches to the problem are complementary since the 
equilibrium canonical distribution containing the specific interaction model for the system may be used to calculate both, the equilibrium free energy and the corresponding FokkerPlanck equation for the non-equilibrium case. $\underline{1,2,7}$

In general, the results obtained by these two approaches are different even if the same interaction model is assumed. To take an example, for the Maier-Saupe interaction model in the uniaxial case, which introduces a parameter $U$ measuring the degree of coupling of one molecule with the average system surrounding it, the equilibrium approach leads to an expression of the free energy in which the dependence on $U$ enters through one term (see, for instance, Eq. (20) of Ref. [19]). If entropic effects are adequately taken into account, other terms of this free energy appear which are independent of the strength of interaction $\underline{\underline{19}}$ In contrast, for this model the dynamic approach leads to expressions of a function playing the role of a free energy in which the parameter $U$ multiplies several terms that cannot be reduced to that of the equilibrium approach (see, for instance, Eqs. (36) and (37) of Ref. [18], among others). In fact, the expression of this function depends on the way in which the hierarchy of equations is closed. This situation is usual in non-equilibrium statistical mechanics when dealing with closure problems. Different approximations may have advantages under different physical conditions.

We may attribute these differences between free energies derived from equilibrium and dynamic approaches to the fact that dynamic closures may incorporate the effect of the relaxation of, in principle, all the moments of the distribution function. As a consequence of this, it is important to check the performance of the obtained results by following general criteria, such as those proposed in Ref. [18]. In addition, because the equilibrium approach is 
general, it seems appropriate to establish a distinction by referring to a free energy when it is derived from equilibrium schemes and to a free-energy like function when the corresponding quantity is derived from dynamic calculations. $\underline{2} \cdot \underline{18}$

Here, we concentrate our analysis on the dynamic closure approach for uniaxial systems and its coupling with the evolution equation of system's mass density. This non-homogenous problem is less studied in the literature despite its great interest because it allows to analyze the physical conditions in which an appropriate perturbation may produce patterns and/or traveling structures in the system. A question of great technological interest.

The existence of these spatial and temporal structures is analyzed by first using, in Section II, the Gibbs entropy postulate 19,22 and the rules of mesoscopic non-equilibrium thermodynamics $22-25$ in order to derive a Fokker-Planck equation governing the evolution of the non-homogeneous distribution of particle orientations. In section III, this equation is used in turn to derive the evolution equations of the second and fourth order orientational tensor parameters in the homogeneous case. Then, the dynamic closure problems addressed in Section IV, where we consider a recent approach $\frac{18}{}$ to determine the forth order scalar parameter as a function of the second order one and then obtain the corresponding evolution equation for the scalar second order parameter. We also consider an approximation of the dynamic equations for both scalar orientational order parameters that allows to propose a novel closure relation leading to a novel expression for the free-energy like function. We test this closure in comparison with those proposed in Refs. [18] and [19] by analyzing its performance under equilibrium and flow conditions. In Section V we derive the non-homogeneous evolution equation for system's density and coupling parameter, and for the scalar second 
order parameter. Using the mentioned closures, we make a linear stability analysis and search for the conditions in which patterns and traveling structures appear. Finally, the main conclusions are presented in Section VI.

\section{DERIVATION OF THE ORIENTATIONAL FOKKER-PLANCK EQUATION}

We shall consider a system of $N$ elongated uniaxial molecules in a thermal bath. The state of each molecule is specified by the position of its center of mass, $\vec{r}$, and by the unitary orientational degree of freedom, $\vec{u}$, associated with its long axis. The dynamics of the system can be described in terms of the single-particle distribution function $f(\vec{r}, \vec{u}, t)$ that is a conserved quantity and evolves in time by following the continuity equation

$$
\frac{\partial f}{\partial t}=-\nabla_{i}\left(f V_{i}\right)-\hat{R}_{i}\left(f \Omega_{i}\right)
$$

where, $V_{i}$ and $\Omega_{i}$ are the conjugate velocities to $x_{i}$ and $u_{i}$, respectively. Here, $\nabla_{i}$ is the $i$-th spatial derivative and $\hat{R}_{i}$ is the $i$-th component of the rotational operator

$$
\hat{R}_{i}=\varepsilon_{i j k} u_{j} \frac{\partial}{\partial u_{k}}
$$

Here, $\varepsilon_{i j k}$ represents the Levi-Civitta antisymmetric symbol and summation over repeated indexes will be implicit in through out this paper.

We will adopt a mean-field approach in which interactions of a single molecule with other molecules are represented by the mean-field potential $\mathcal{U}_{\mathrm{mf}}$ that can be a function of both position and orientation vectors, i.e. $\mathcal{U}_{\mathrm{mf}}=\mathcal{U}_{\mathrm{mf}}(\vec{r}, \vec{u})$. In addition, we will also consider an external non-homogeneous time-dependent force characterized by the potential $\mathcal{U}_{\mathrm{e}}$ that acts on the ensemble of molecules and drives it out from equilibrium. Such a force may represent, 
for instance, the influence of an electric field. $\underline{15}, \underline{17}, \underline{24} \underline{\underline{26}}$ The spatial and temporal variations of the total potential field $\mathcal{U}=\mathcal{U}_{\mathrm{mf}}+\mathcal{U}_{\mathrm{e}}$ are assumed to evolve in space and time-scales large as compared to the time-scales of the relaxation of fluctuations. Accordingly, we assume the existence of a local-equilibrium probability distribution, $f^{\text {leq }}(\vec{r}, \vec{u} ; t)$, which is defined in terms of the previous potential by the canonical relation

$$
f^{\text {leq }}(\vec{r}, \vec{u}, t)=\frac{1}{z^{\text {leq }}(T ; t)} \exp \left[-\frac{\mathcal{U}(\vec{r}, \vec{u}, t)}{k_{B} T}\right]
$$

where $z^{\text {leq }}$ is the corresponding partition function, $T$ is the temperature and $k_{B}$ is the Boltzmann constant. The particular expression for $f^{\text {leq }}$ can be obtained from equilibrium calculations.,$\frac{11,19,20}{1}$

The explicit form of Eq. (1) in terms of $f(\vec{r}, \vec{u}, t)$ can be obtained by calculating the rate of entropy production of the system during its evolution in time with the help of the Gibbs

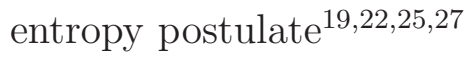

$$
\mathcal{S}=-k_{B} \iint d \vec{r} d \vec{u} f(\vec{r}, \vec{u}, t) \ln \left[\frac{f(\vec{r}, \vec{u}, t)}{f^{\text {leq }}(\vec{r}, \vec{u} ; t)}\right]+\mathcal{S}^{\text {leq }},
$$

where $\mathcal{S}^{\text {leq }}$ is the entropy of the local-equilibrium state. The entropy production rate $\sigma=$ $\partial\left(\mathcal{S}-\mathcal{S}^{\text {leq }}\right) / \partial t$, is therefore obtained by calculating the time derivative of Eq. (2), and using Eq. (11). This procedure yields

$$
\sigma=\frac{1}{T} \iint d \vec{r} d \vec{u}\left[\mu \nabla_{i}\left(f V_{i}\right)+\mu \hat{R}_{i}\left(f \Omega_{i}\right)\right]+k_{B} \iint d \vec{r} d \vec{u} f \frac{\partial}{\partial t} \ln f^{\text {leq }},
$$

where the non-equilibrium chemical potential, $\mu=\mu(\vec{r}, \vec{u}, t)$, has been defined by

$$
\Delta \mu(\vec{r}, \vec{u}, t)=k_{B} T \ln \left[\frac{f(\vec{r}, \vec{u}, t)}{f^{\text {leq }}(\vec{r}, \vec{u}, t)}\right] .
$$


As we mentioned before, changes in time of the local equilibrium distribution can be neglected in the limit of slow external perturbations and, consequently, the second term on the right hand side of Eq. (4) vanishes. In this case, Eq. (44) takes the form

$$
\sigma=-\frac{1}{T} \iint d \vec{r} d \vec{u}\left(f V_{i} \nabla_{i} \mu+f \Omega_{i} \hat{R}_{i} \mu\right)
$$

where we have performed an integration by parts and assumed an infinite system with vanishing probability density at the boundaries in $\vec{r}$-space as well as periodic conditions in $\vec{u}$-space.

The entropy production rate given by Eq. (66) has the form of a sum of products of generalized currents, $V_{i}$ and $\Omega_{i}$, with generalized forces, $\nabla_{i} \mu$ and $\hat{R}_{i} \mu$. According to the rules of mesoscopic non-equilibrium thermodynamics, we may follow a linear response scheme in which currents are proportional to forces $\underset{22}{2,28}$ Thus, the currents $V_{i}$ and $\Omega_{i}$ are given in terms of $\nabla_{i} \mu$ and $\hat{R}_{i} \mu$ by the relations

$$
\begin{gathered}
V_{i}=-K_{i j} \nabla_{j} \mu-M_{i j} \hat{R}_{j} \mu, \\
\Omega_{i}=-L_{i j} \hat{R}_{j} \mu-\tilde{M}_{i j} \nabla_{j} \mu,
\end{gathered}
$$

where $K_{i j}, L_{i j}$ and $M_{i j}$ are Onsager coefficients with $M_{i j}=-\tilde{M}_{i j}$. Replacing Eqs. (7) and (8) into Eq. (11), using the expressions for the local equilibrium distribution (2) and the non-equilibrium chemical potential (5), we finally obtain a closed differential equation for $f$ :

$$
\frac{\partial f}{\partial t}=\nabla_{i}\left[D_{i j}\left(\nabla_{j} f+\frac{f}{k_{B} T} \nabla_{j} \mathcal{U}\right)\right]+\hat{R}_{i}\left[\mathcal{D}_{i j}\left(\hat{R}_{j} f+\frac{f}{k_{B} T} \hat{R}_{j} \mathcal{U}\right)\right] .
$$

Here, we have introduced the diffusion tensors $D_{i j}=k_{B} T K_{i j}$ and $\mathcal{D}_{i j}=k_{B} T L_{i j}$. For anisotropic molecules both rotational and translational diffusion tensors depend on the orientational degrees of freedom $u_{i}$. The translational diffusion tensor can be expressed in terms 
of the parallel $D_{\|}$and perpendicular $D_{\perp}$ (to the symmetry axis) coefficients in the form $\underline{\underline{1}}$

$$
D_{i j}=D_{\|} u_{i} u_{j}+D_{\perp}\left(\delta_{i j}-u_{i} u_{j}\right)=\bar{D} \delta_{i j}+D_{a}\left(u_{i} u_{j}-\frac{1}{3} \delta_{i j}\right)
$$

where we have defined $\bar{D}=\left(D_{\|}+2 D_{\perp}\right) / 3$ and $D_{a}=D_{\|}-D_{\perp}$.

At mesoscopic level, the FPE Eq. (9) provides the complete dynamical description of an ensemble of uniaxial molecules subjected to mean-field and external forces. It coincides with the ones derived in Refs. [1] and [14] and therefore shows their compatibility with the second law of thermodynamics. A detailed analysis of the relation of this equation with its counterpart derived from kinetic theory arguments in different physical situations can be found in Refs. [2] and [21]. In addition it is convenient to mention that the present formalism can be generalized to consider the effect of the relaxation of the fluctuating velocities (angular and translational) of the molecules in the macroscopic relaxation of the system. This may lead to non-trivial diffusion effects, especially in the case of flowing systems. $\underline{29}, \underline{30}$

\section{HIERARCHY OF MACROSCOPIC DYNAMIC EQUATIONS}

Experimental data characterizing the behavior of a system is more frequently obtained through the time course of the components of the tensor order parameter and higher order moments of the distribution function $f$, than from the form and behavior of the distribution itself. Thus, it is convenient to derive the evolution equations for these moments. For simplicity, this will be done for the case when non-homogeneities can be neglected and therefore we shall assume that no external fields are applied and that the description can be 
carried out in terms of the reduced probability density

$$
g(\vec{u}, t)=\int d \vec{r} f(\vec{r}, \vec{u}, t)
$$

which obeys the reduced FPE

$$
\frac{\partial g}{\partial t}=\hat{R}_{i}\left[\mathcal{D}_{i j}\left(\hat{R}_{j} g+\frac{g}{k_{B} T} \hat{R}_{j} \mathcal{U}\right)\right]
$$

Here, we will assume that each molecule evolves in the presence of a Maier-Saupe mean-field potential of the form ${ }^{1,2}$

$$
\mathcal{U}_{\mathrm{mf}}=-\frac{3}{2} U k_{B} T u_{i} u_{j} S_{i j}
$$

where $S_{i j}$ is the second order orientational tensor whose mathematical definition is given in Eq. (14) below. In addition, $U$ is a parameter that measures the degree of coupling of the particle with its surroundings and depends on the molecular structure and interactions $\underline{31}$ For a concentrated solution of rigid rodlike polymers of length $L$ and diameter $b, U$ is proportional to $\rho b L$, where $\rho$ is the number of polymers per unit volume. $\underline{2}^{2}$

Following Ref. [1], the moments of $g$ are defined through symmetric traceless tensors of rank two, four, etc., corresponding to a multipolar expansion of the orientational degree of freedom. In the next, the dyad product of a vector, $\vec{u}$, with itself $n$ times, will be denoted by $\mathbf{u}_{(n)}$ and $\mathbf{1}$ will denote the unitary tensor of rank two. General tensors will be denoted by blackboard bold characters, $\mathbb{A}, \mathbb{B}$, etc. Indices notation will be also used when convenient. Finally, $[\mathbb{A}]^{s}$ will denote the symmetric part of the tensor $\mathbb{A}$.

The symmetric traceless tensors of rank two, four and six, are respectively defined by the following averages over the distribution $g, \underline{1}$

$$
\mathbb{S}=\left\langle\mathbf{u}_{(2)}-\frac{1}{3} \mathbf{1}\right\rangle
$$




$$
\begin{gathered}
\mathbb{W}=\left\langle\mathbf{u}_{(4)}-\frac{6}{7}\left[\mathbf{u}_{(2)} \mathbf{1}\right]^{s}+\frac{3}{35}[\mathbf{1 1}]^{s}\right\rangle \\
\mathbb{Z}=\left\langle\mathbf{u}_{(6)}-\frac{15}{11}\left[\mathbf{u}_{(4)} \mathbf{1}\right]^{s}+\frac{15}{33}\left[\mathbf{u}_{(2)} \mathbf{1 1}\right]^{s}-\frac{5}{231}[\mathbf{1 1 1}]^{s}\right\rangle .
\end{gathered}
$$

Following an usual scheme in studying the mesoscopic dynamics of nematic liquid crystals, $\underline{\underline{2}}$ we shall assume that rotational diffusion is isotropic, that is, $\mathcal{D}_{i j}=\mathcal{D} \delta_{i j}$ with $\mathcal{D}$ a constant. This assumption is consistent with the preaveraging approximation where a constant preaveraged rotational diffusion coefficient is introduced., $, \underline{16}, \underline{32}$

Thus, using the reduced FPE Eq. (12), and the previous definitions, the explicit evolution equations obtained for $\mathbb{S}$ and $\mathbb{W}$ are

$$
\begin{aligned}
\frac{\partial}{\partial t} \mathbb{S}= & -6 \mathcal{D} \mathbb{S}+6 \mathcal{D} U\left[\frac{1}{5} \mathbb{S}+\frac{3}{7}\left(\mathbb{S} \cdot \mathbb{S}-\frac{1}{3} \mathbb{S}: \mathbb{S} \mathbf{1}\right)-\mathbb{W}: \mathbb{S}\right] \\
\frac{\partial}{\partial t} \mathbb{W}= & -20 \mathcal{D} \mathbb{W}+\frac{4}{49} \mathcal{D} U\left(35[\mathbb{S S}]^{s}+2 \mathbb{S}: \mathbb{S}[\mathbf{1 1}]^{s}-20[\mathbb{S} \cdot \mathbb{S} \mathbf{1}]^{s}\right) \\
& +\frac{36}{11} \mathcal{D} U\left([\mathbb{S} \cdot \mathbb{W}]^{s}-\frac{3}{7}[\mathbb{S}: \mathbb{W} \mathbf{1}]^{s}\right)-12 \mathcal{D} U \mathbb{Z}: \mathbb{S} .
\end{aligned}
$$

Equations (17) and (18) are the first two of an infinite hierarchy of coupled differential equations for the moments of the orientational distribution. It is important to notice that, in general, the evolution equation for the moment of order $n$ has terms containing the moments of order $n-2$ and $n+2$, thus coupling the whole hierarchy of equations. This is a well known result that is explained in whole detail in the classical textbook by Kröger where the complete hierarchy is explicitly calculated. $\underline{\underline{1}}$

An important fact to mention is that the characteristic relaxation time for each one of the equations in this hierarchy is proportional to $\mathcal{D}^{-1}$. As a consequence of this, it is not possible to give a general criterium based on time scales to cut the hierarchy of equations 
and search for consistent dynamic closures. Due to this fact, many dynamic closures to the problem have been proposed in the literature to cut the hierarchy. The validity and physical consistency of the most representative of them has been analyzed in detail in Ref. [18]. However, for comparison purposes, let us recall the classical decoupling approximation proposed in Refs. [1, 2, 7], where

$$
\left\langle\mathbf{u}_{(4)}\right\rangle: \mathbb{S}=\left\langle\mathbf{u}_{(2)}\right\rangle\left\langle\mathbf{u}_{(2)}\right\rangle: \mathbb{S} .
$$

In terms of the tensors $\mathbb{S}$ and $\mathbb{W}$, the previous relation can be written in the form

$$
\mathbb{W}: \mathbb{S}=-\frac{2}{15} \mathbb{S}-\frac{4}{7} \mathbb{S} \cdot \mathbb{S}+\mathbb{S}: \mathbb{S} \mathbb{S}+\frac{4}{21} \mathbb{S}: \mathbb{S} \mathbf{1}
$$

Indeed, by replacing Eq. (19) into Eq. (17), we recover the well known closed expression for the order parameter of the Doi-Hess model

$$
\frac{\partial}{\partial t} \mathbb{S}=-6 \mathcal{D}\left[\left(1-\frac{U}{3}\right) \mathbb{S}-U\left(\mathbb{S} \cdot \mathbb{S}-\frac{1}{3} \mathbb{S}: \mathbb{S} \mathbf{1}\right)+U \mathbb{S}: \mathbb{S} \mathbb{S}\right]
$$

This approximation, as the other ones analyzed in Ref. [18], truncates the infinite hierarchy of equations for the moments of the distribution to the lowest possible order. An important fact to mention here is that the last three terms at the right hand side of the previous equation are proportional to the coupling parameter $U$. As mentioned in the introduction, this is a characteristic result of the dynamic closures in uniaxial systems. The corresponding free-energy like function that may be obtained from it by setting the time derivative equal to zero, will contain only one term independent of $U$, which arises from the first term in (20) and is related to the entropic force term of the reduced Fokker-Planck equation (12) ${ }^{2}$ 


\section{DYNAMIC CLOSURES FOR THE EVOLUTION EQUATIONS OF THE SCALAR ORDER PARAMETERS}

We shall consider here a uniaxial nematic liquid crystal. For simplicity, it will be assumed to be initially oriented by an external field along the unitary vector $\vec{n}$, which is called the director. In the following, the dynamics of this phase will be described in terms of the scalar order parameters $S, W$ and $Z$, which are respectively defined in terms of $\vec{n}$ and the order parameters tensors $\mathbb{S}, \mathbb{W}$ and $\mathbb{Z}$, Eqs. (14)-(16), by ${ }^{\underline{1}}$

$$
\begin{gathered}
S=\frac{3}{2} n_{i} S_{i j} n_{j}=\left\langle P_{2}(\vec{u} \cdot \vec{n})\right\rangle, \\
W=\frac{35}{8} n_{i} n_{j} W_{i j k l} n_{k} n_{l}=\left\langle P_{4}(\vec{u} \cdot \vec{n})\right\rangle, \\
Z=\frac{231}{16} n_{i} n_{j} n_{k} Z_{i j k l p q} n_{l} n_{p} n_{q}=\left\langle P_{6}(\vec{u} \cdot \vec{n})\right\rangle,
\end{gathered}
$$

where $P_{m}$ is the Lengendre polynomial of order $m$. Notice that $S, W$, and $Z$ are zero in the isotropic limit and 1 in the completely ordered phase.

In terms of $S, W$ and $Z$, the following closure relation can be written for $\mathbb{Z}$ in terms of $\mathbb{S}$ and $\mathbb{W}$,

$$
\mathbb{Z}=\frac{Z}{S W}\left\{[\mathbb{W} \mathbb{S}]^{s}-\frac{8}{11}[\mathbb{S} \cdot \mathbb{W} \mathbf{1}]^{s}+\frac{4}{33}[\mathbb{S}: \mathbb{W} \mathbf{1} 1]^{s}\right\},
$$

where the right hand side contains the symmetric traceless part of the product of $\mathbb{W} \mathbb{S}$. This explicit relation representing $\mathbb{Z}$ is indeed a particular case of the general closure equation for moments of arbitrary order derived in Ref. $\underline{18}$.

Using Eq. (24) the evolution equations for the first two scalar moments can be obtained 
by projecting Eqs. (14) and (15) on $\vec{n}$. The result of this procedure is

$$
\begin{gathered}
\frac{\partial S}{\partial t}=-6 \mathcal{D}\left(1-\frac{U}{5}\right) S+\frac{6}{7} \mathcal{D} U S^{2}-\frac{72}{35} \mathcal{D} U S W \\
\frac{\partial W}{\partial t}=-20 \mathcal{D} W+\frac{20}{7} \mathcal{D} U S^{2}+\frac{60}{77} \mathcal{D} U S W-\frac{40}{11} \mathcal{D} U S Z .
\end{gathered}
$$

Notice that an infinite hyerarchy of coupled equations for the scalar moments is obtained, which must be closed at a certain level. In the subsequent sections, two different closure relations will be discussed.

\section{A. Parametric closure}

With the purpose of closing the infinite hyerarchy of coupled equations for the scalar order parameters, approximate relationships between $S, W$ and $Z$ can be obtained based on the uniaxial orientational distribution of the Maier-Saupe type $\mathrm{18}^{18}$

$$
g(\vec{u})=G \exp [a \vec{u} \cdot \mathbb{A} \cdot \vec{u}]
$$

which is a special case of the Bingham distribution. ${ }^{20}$ Here, $a$ is a parameter, $\mathbb{A}$ is a symmetric traceless matrix and $G$ is the normalization constant.

Using Eq. (27), $S, W$ and $Z$ can be calculated in terms of $a$, from which the numerical

relation between these quantities can be obtained. From this analysis it has been shown in Ref. $\stackrel{11,18}{1}$ that $W$ and $Z$ can be very well approximated in terms of $S$ by

$$
W=S\left(1-(1-S)^{\nu}\right)
$$

with $\nu=3 / 5$; and

$$
Z=S^{6},
$$


respectively. The first expression can be used in Eqs. (25) and (26) in order to close the hyerarchy of equations upto order $S$, while the latter allows for closing it at order $W$.

Equation (28) will be referred hereafter as the parametric closure. It has been shown to fullfill the constraints imposed by the nematic symmetry and to be correct in the isotropic and totally aligned cases. It also yields the following evolution equation for $S$

$$
\frac{\partial S}{\partial t}=-6 \mathcal{D}\left[\left(1-\frac{U}{5}\right) S-\frac{U}{7} S^{2}+\frac{12}{35} U S^{2}\left(1-(1-S)^{\nu}\right)\right]
$$

Dynamic equations of the form of the previous one, have been extensively used in the literature of the field since the pioneer works of Doi and Hess in order to identify a function $A=A(U, S)$ through the phenomenological relation ${ }^{2}, \underline{18}$

$$
\frac{\partial S}{\partial t}=-L \frac{\partial A}{\partial S}
$$

such that $A$ plays the role of a free energy. As we mentioned previously, it is convenient to refer to this quantity as a free-energy like function since it differs from those calculated following equilibrium techniques. In the present case, the dynamic closure was implemented by calculating the equilibrium averages of the second and fourth order orientational parameters, and using an interpolation function directly in the evolution equation for the second order orientational parameter $\stackrel{18}{1}$ In the following subsection, we will show how the dynamic closure approximation is modified when the relaxation of the fourth order orientational parameter is also taken into account.

For the parametric model, $A$ has the explicit form originally obtained in Ref. $\underline{18}$

$$
A=\frac{1}{2}\left(1-\frac{U}{5}\right) S^{2}+\frac{U}{15} S^{3}+\frac{12 U\left(1-S^{1+\nu}\right)\{2+S(1+\nu)[2+S(2+\nu)]\}}{35(1+\nu)(2+\nu)(3+\nu)}
$$


and predicts that for $U<U_{1}^{*}=4.48, A$ has only one minimum at $S=0$, corresponding to the isotropic phase. For $U_{1}^{*}<U<U_{2}^{*}=5, A$ has two minima, one at $S=0$ and the other one at $S>0$, indicating that the system can be found in the nematic or the isotropic phase depending on the initial value of $S$. For $U \geq U_{2}^{*}, A$ has a local maximum at $S=0$, and the nematic phase is the stable one.

The equilibrium values of the order parameter can be found from the condition

$$
\left.\frac{\partial A}{\partial S}\right|_{S_{\mathrm{eq}}}=0,
$$

from which it follows that $S_{\text {eq }}\left(U_{1}^{*}\right)=0.31$.

It should be stressed that although the function $A$ given by Eq. (32) exhibits the typical behavior used for describing the I-NPT, it is not completely consistent as long as it is finite at $S=1$, thus allowing for $S$ to take nonphysical values, i.e. $S>1$ for finite energies. This problem has been successfully solved in Ref. $\underline{\underline{19}}$, where a thermodynamic free-energy has been derived by maximizing the Gibbs entropy postulate (3) with the isotropic state as the reference one. The obtained expression ensures the constraint $S<1$ for arbitrary energies.

Concerning the high order closure relation (29), it should be mentioned that when it is replaced into Eqs. (25) and (26), and the resulting closed system is used to find the equilibrium values of the order parameter $S$ by imposing static conditions, i.e. $\partial S / \partial t=$ $\partial W / \partial t=0$, then nonphysical behavior is observed in $A$ since $S_{\text {eq }}$ turns out to be a decreasing function of $U$ for relative large values of this quantity. Therefore, it should be remarked that although Eq. (29) can be used to approximate very well the values of $Z$, it can not be used consistently in the dynamic equations for $S$ and $W$ by imposing the static conditions already mentioned. This suggests that having expressions for higher order moments which 
approximate very well their exact values, might not be sufficient to fullfill the requirements imposed by the dynamic equations.

\section{B. Dynamic closure}

Here we shall introduce an alternative closure relation for $W$ in terms of $S$, which has the explicit dependence

$$
W=S^{\frac{10}{3}},
$$

and will be referred hereafter as the dynamic closure relation since it is motivated by the explicit form of the evolution equations for $S$ and $W$, Eqs. (25) and (26). Indeed, it can be verified that Eq. (34) is exact in two important limiting cases, namely: for $U \ll 1$, i.e. in the isotropic phase; and for $U \gg 1$, i.e. close to the completely aligned phase.

Consequently, this new closure relation is not intended to be exact, but it is an interpolating expression which is consistent with the time evolution of the scalar order parameters. Furthermore, it turns out to approximate the correct parametric relation within the same accuracy degree than Eq. (28). This is explicitely shown in the left panel of Fig. 1, where the deviations from the closure relations Eqs. (28) and (34) from the exact numerical parametric value of $W$ are presented. It can be noticed that the maximum deviation observed for the parametric closure, Eq. (28), is 0.052; while the corresponding value for the dynamic approximation, Eq. (34), is 0.065. Notice also that the parametric closure exhibits its maximum deviations in the region $S \simeq 1$, where the dynamic closure approximates $W$ better. 

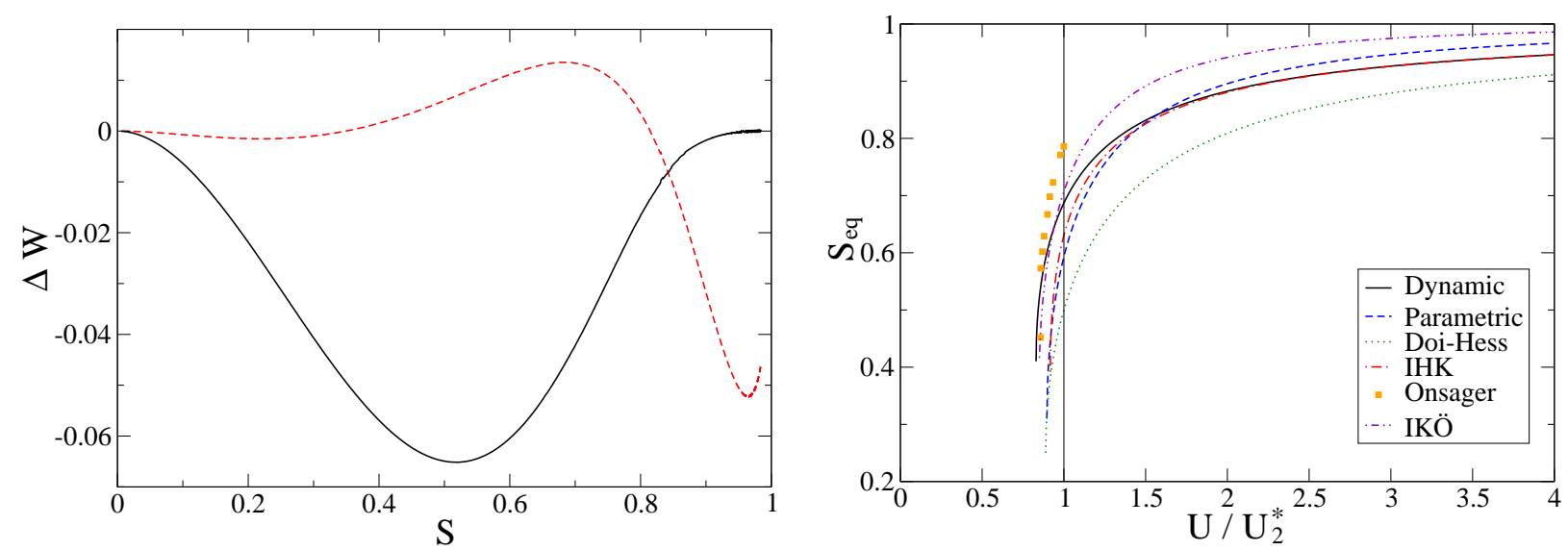

FIG. 1: Left panel: Performances of the parametric closure, Eq. (28) (dashed line), and the dynamic closure, Eq. (34) (solid line). $\Delta W$ represents the difference of the approximated expressions (28) and (34), with respect to the numerical value of the order parameter $W$ obtained from the orientational distribution (27). Right panel: Equilibrium values of the order parameter, $S_{\text {eq }}$, for the nematic phase. The black solid line corresponds to the dynamic closure (36), which incorporates the effects of the relaxation dynamics of $S$ and $W$. Orange square symbols were taken from Ref. [14] and correspond to the numerical solution of the Onsager model. The red dashed-dotted line corresponds to the IHK model ${ }^{19}$ that incorporates entropic effects in the Maier-Saupe potential whereas the blue dashed line comes from the $(\mathrm{KAC})^{18}$ parametric closure (28). IKÖ model 14 is represented by the purple dashed double-dotted line and finally the green dotted line represents the classical solution obtained from Doi-Hess model. $\underline{2}$

The dynamic closure relation, Eq. (34), yields the following dynamic equation for $S$,

$$
\frac{\partial S}{\partial t}=-6 \mathcal{D}\left[\left(1-\frac{U}{5}\right) S-\frac{U}{7} S^{2}+\frac{12}{35} U S^{\frac{13}{3}}\right]
$$




\begin{tabular}{|c|c|c|c|c|c|}
\hline Model & Equilibrium (IHK) & Parametric (KAC) & Dynamic (HMS) & Doi & IKÖ \\
\hline \hline$U_{1}^{*}$ & 4.59 & 4.48 & 4.15 & 2.67 & 6.22 \\
\hline$U_{2}^{*}$ & 5.0 & 5.0 & 5.0 & 3.0 & 7.34 \\
\hline$S_{\text {eq }}\left(U_{1}^{*}\right)$ & 0.39 & 0.31 & 0.41 & 0.25 & 0.37 \\
\hline
\end{tabular}

TABLE I: Parameters characterizing the I-NPT in diverse models. $U_{1}^{*}$ represents the smallest value of $U$ at which the nematic phase can be observed, while $U_{2}^{*}$ is the strength of the mean field interaction at which the isotropic phase becomes unstable. $S_{\text {eq }}\left(U_{1}^{*}\right)$ is the equilibrium value of the order parameter at the I-NPT. This comparison can be extended by considering Ref $\underline{18}$, where an extensive study which includes more models can be found.

and the corresponding function $A=A(U, S)$ for this approximation reads

$$
A=\frac{1}{2}\left(1-\frac{U}{5}\right) S^{2}-\frac{U}{21} S^{3}+\frac{9}{140} U S^{\frac{16}{3}}
$$

which predicts the values of the parameters characterizing the I-NPT $U_{1}^{*}, U_{2}^{*}$ and $S_{\text {eq }}\left(U_{1}^{*}\right)$, shown in Table 1, where these parameters are compared with those obtained from other models including the parametric closure.

The equilibrium values of the scalar order parameter in the nematic phase for the dynamic closure can be found from Eqs. (33) and (36). The solution is shown on the right panel of Figure 1 as function of the reduced interaction strength $U / U_{2}^{*}$. Figure 1 also shows $S_{\text {eq }}$ for other models including the classical model of Doi and Hess 2 ; the numerical solution of the Onsager excluded volume theory as it appears in Ref. [14]; the model proposed in Ref. 14 by Ilg, Karling and Ötinger (IKÖ), where a generalized mean-field interaction is introduced; and the solution obtained from the parametric KAC closure Eq. (28). It can be observed 

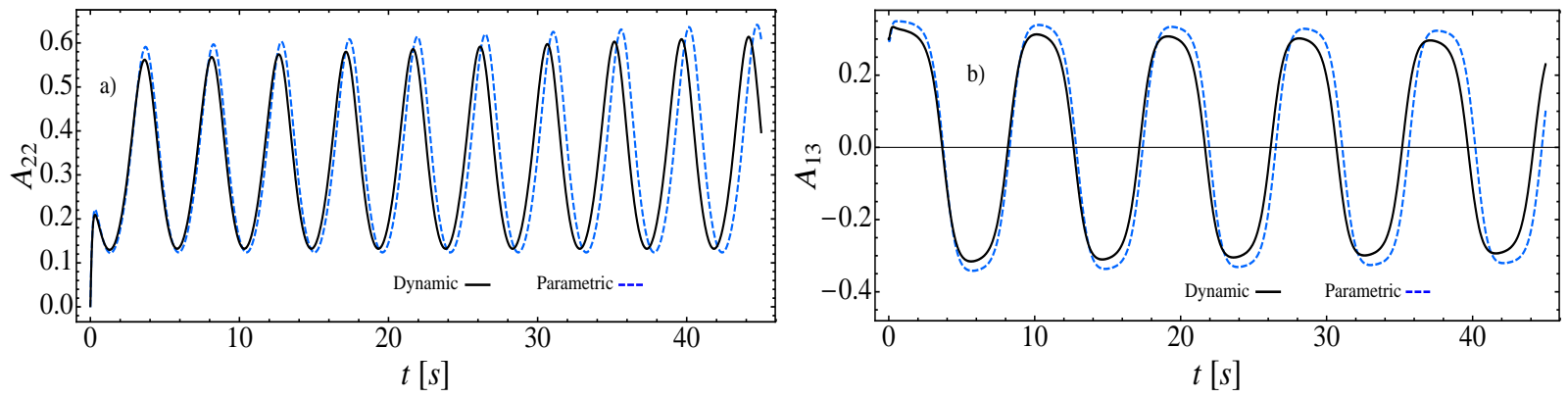

FIG. 2: Oscillations of two elements of the tensor $\mathbb{A}$ in terms of time obtained after numerically solving Eq. (38). In a) we present the diagonal component $A_{22}$, whereas in b) the component $A_{13}$ is shown. The values of the parameters used were $\mathcal{D}=0.3, \mathrm{U}=9, Q=1.5$ and the magnitude of the shear rate $|\nabla \vec{v}|=1.5$.

that our model predicts a wider range of values of $U$ for the coexistence of the isotropic and nematic phases than all the other models. Finally, Figure 3 shows the bifurcation diagram obtained from the parametric and the dynamic models.

\section{Performance of the parametric and dynamic closures under flow conditions}

In order to rigorously test the performance of the novel closure approximation Eq. (34), we will study its behavior under flow conditions. For simplicity, we will restrict our analysis to a comparison with respect to the performance of the parametric closure Eq. (28), which in turn has been compared with several closure models as well as with exact results obtained from the numerical solution of the FPE $\stackrel{18}{\underline{-}}$ Our comparison is justified since in the latter case it has shown that Eq. (28) is valid with high precision for a wide range of conditions.

We thus consider an elongated molecule immersed in a fluid with a velocity gradient and 


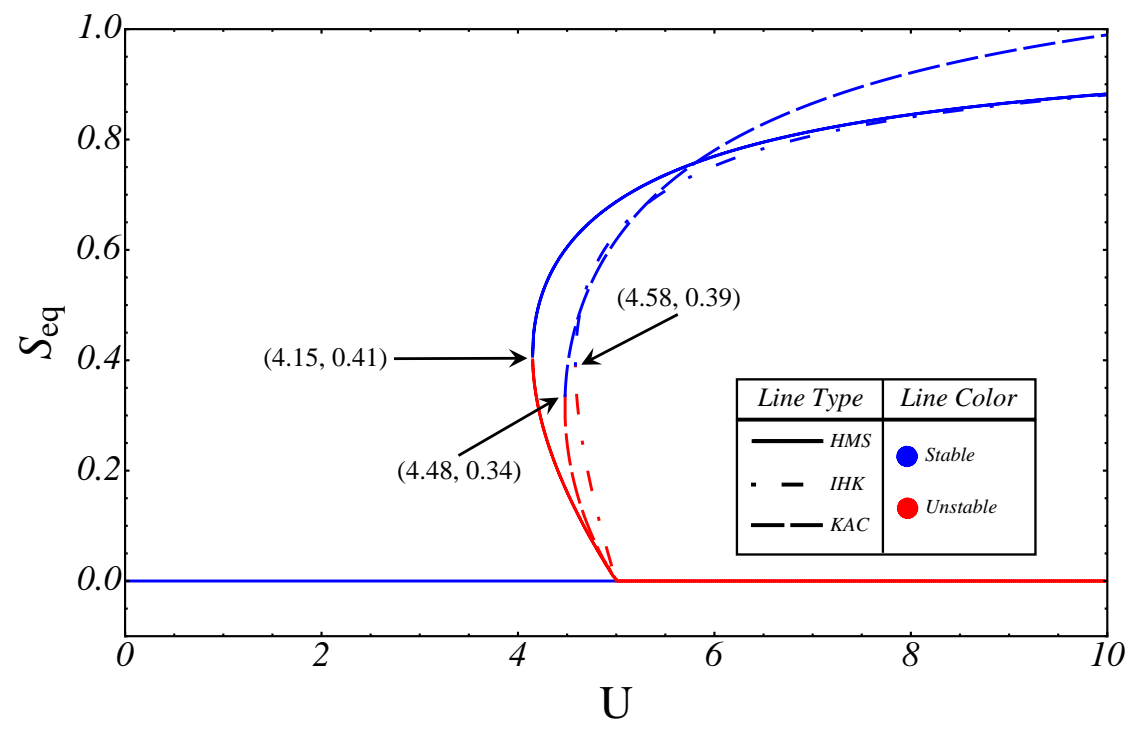

FIG. 3: Bifurcation diagram of the scalar order parameter $S_{\text {eq }}$ as a function of the interaction strength $U$, as obtained from the parametric and the dynamic closures, Eqs. (28) (denoted by KAC, dashed line) and (34) (denoted by HMS, solid line). We also included the equilibrium closure reported in Ref. $\underline{\underline{19}}$ (denoted by IHL, dash-dotted line). The blue portion of the lines indicates stable states whereas the red portion unstable states. The purple portion indicates a stable region for KAC and HMS, and unstable for IHK.

subject to the potential $\mathcal{U}$. The FPE corresponding to this problem has been calculated by considering the hydrodynamic torques which the fluid exerts on the molecule. $\underline{2}, \underline{7}$ This calculation yields

$$
\frac{\partial g}{\partial t}=-\omega_{i} \hat{R}_{i} g-\frac{1}{2} P \hat{R}_{i}\left[g \hat{R}_{i}\left(u_{j} u_{k} K_{j k}\right)\right]+\hat{R}_{i}\left[\mathcal{D}_{i j}\left(\hat{R}_{j} g+\frac{g}{k_{B} T} \hat{R}_{j} \mathcal{U}\right)\right]
$$

where $\omega_{i}=\frac{1}{2} \varepsilon_{i j k} \nabla_{j} v_{k}$ is the vorticity, $K_{i j}=\frac{1}{2}\left(\nabla_{i} v_{j}+\nabla_{j} v_{i}\right)$ is the symmetric part of the velocity gradient, and $P=\left(Q^{2}-1\right) /\left(Q^{2}+1\right)$ is the shape factor of an ellipsoid of revolution with axis ratio $Q$. 
Once again, we will consider only a mean field potential of the Maier-Saupe type, Eq. (13), and calculate the evolution equation for the second order tensor, $\mathbb{A}=\left\langle\mathbf{u}_{(2)}\right\rangle=\mathbb{S}+\frac{1}{3} \mathbf{1}$, in the presence of flow directly from Eq. (37). This equation involves the fourth order moment $\mathbb{B}=\left\langle\mathbf{u}_{(4)}\right\rangle$, and explicitly reads

$$
\begin{aligned}
\frac{\partial}{\partial t} \mathbb{A}+\vec{v} \cdot \nabla \mathbb{A}= & \mathbb{A} \cdot \mathbb{L}-\mathbb{L} \cdot \mathbb{A}+P(\mathbb{A} \cdot \mathbb{K}+\mathbb{K} \cdot \mathbb{A})-2 P \mathbb{K}: \mathbb{B} \\
& -6 \mathcal{D}\left(\mathbb{A}-\frac{1}{3} \mathbf{1}\right)+6 \mathcal{D} U(\mathbb{A} \cdot \mathbb{A}-\mathbb{A}: \mathbb{B}),
\end{aligned}
$$

where $L_{i j}=\frac{1}{2}\left(\nabla_{i} v_{j}-\nabla_{j} v_{i}\right)$ is the antisymmetric part of the velocity gradient.

This equation can be closed by using the consistent closure relationship between $\mathbb{A}$ and $\mathbb{B}^{18}$

$$
\mathbb{B}=\alpha[\mathbb{A}]^{s}-2 \beta[\mathbb{A} \mathbf{1}]^{s}-2 \gamma[\mathbf{1 1}]^{s},
$$

where the coefficients $\alpha, \beta$ and $\gamma$ depend on the scalar order parameters $S$ and $W$ through

$$
\begin{gathered}
\alpha=\frac{W}{S^{2}} \\
\beta=\frac{\alpha}{3}+\frac{2 W}{21 S}-\frac{3}{7} \\
\gamma=\frac{3}{70}+\frac{2 W}{45}-\frac{\alpha}{18}-\frac{2 W}{63 S}
\end{gathered}
$$

Thus, we replace Eq. (39) into Eq. (38) and solve the result numerically for the components $A_{i j}$. We consider both, the parametric and the dynamic closure approximations, given by Eqs. (28) and (34), respectively, in order to compare their performances. For simplicity, we assume a homogeneous aligment tensor $\mathbb{A}$, and restrict our analysis to situations similar to those reported in the recent literature. Figure 2 summarizes our results. There we present the behavior of two different components of $\mathbb{A}$, obtained for diverse values of the parameters 
$P, \mathcal{D}, U$ and $\nabla \vec{v}$ as indicated in the caption. It can be observed that the dynamic closure relation, Eq. (34), performs quantitatively very well when compared with the parametric closure Eq. (28), and consequently can be also used to approximate the exact solution with high precision at least for the range of values considered here.

\section{PATTERN FORMATION AS A CONSEQUENCE OF THE COUPLING OF THE DENSITY AND THE SCALAR ORDER PARAMETER}

The Fokker-Planck equation derived in Section II and the closure approximations discussed in the previous section can be used to show that patterns and traveling waves may emerge for these systems by following a mechanism different to those discussed previously in the literature, where the effect of an external driving was considered. $\underline{\underline{34}} \underline{\underline{36}}$ These non-equilibrium structures may occur when the parameter $U$ takes values in the range $U_{1}^{*}<U<U_{2}^{*}$, that is, when coexistence of isotropic and nematic phases is possible.

Essentially, the existence of patterns comes from the fact that the degree of coupling $U$ may depend on the number density of molecules $\rho$. For a lyotropic liquid crystal we may write $U=\left(\rho / \rho^{*}\right) U_{0}$, with $U_{0}$ and $\rho^{*}$ the characteristic energy and density of the system,

respectively $\underline{\underline{2}}$ This dependence indicates that, when increasing the density of the system one also increases the interaction energy and the orientational order. This is the basic mechanism leading to the INP-T. The formation of stationary and dynamic patterns comes from the fact that the resulting equations for $S(\vec{r}, t)$ and $\rho(\vec{r}, t)$, or similarly $U(\vec{r}, t)$, constitute a set of two coupled equations of the reaction-diffusion type $\underline{\underline{37}}$

To show this, let us first obtain a dynamic equation for the coupling parameter $U(\vec{r}, t)$, 
introduced in the description through the Maier-Saupe potential, Eq. (13). We start by deriving the dynamic equation for the reduced probability density $\rho$ defined by

$$
\rho(\vec{r}, t) \equiv \int f(\vec{r}, \vec{u}, t) d \vec{u}
$$

Integrating the Fokker-Planck equation (9) over the solid angle yields the following evolution equation for the number density

$$
\frac{\partial \rho}{\partial t}=\bar{D} \nabla^{2} \rho-\frac{1}{2} \bar{D} \nabla^{2}\left(U S^{2}\right)-\frac{1}{2} \bar{D} \nabla \cdot\left[S^{2} \nabla U(\rho)\right]
$$

where we have used Eq. (13) and $\bar{D}$ has been assumed to be constant. Using the relation $\rho=\left(U / U_{0}\right) \rho^{*}$ with $U_{0}=5$ in the left hand side of Eq. (44) and rearranging terms we finally obtain

$$
\frac{\partial U}{\partial t}=\bar{D}\left(1-\frac{5}{\rho^{*}} S^{2}\right) \nabla^{2} U-\frac{15}{\rho^{*}} \bar{D} S[\nabla S \cdot \nabla U]-\frac{5}{2 \rho^{*}} \bar{D} U \nabla^{2} S^{2}
$$

This equation introduces the effective diffusion coefficient $D_{\text {eff }}=\bar{D}\left(1-\frac{5}{\rho^{*}} S^{2}\right)$. It is interesting to notice that the correcting factor implies that when orientational order increases in the system the diffusion of the particles decreases. However, it is worth stress that molecular dynamics simulations predicted that the average diffusion coefficient $\bar{D}$ may increase under this conditions. ${ }^{33}$ This competing interplay may lead to an interesting non-trivial (non-monotonic) behavior of the effective diffusion coefficient.

To complete the description, we may use Eq. (9) to derive a general evolution equation for the non-homogeneous order parameter tensor $S_{i j}(\vec{r}, t)$ defined in Eq. (14). Multiplying Eq. (9) by $u_{i} u_{j}-\frac{1}{3} \delta_{i j}$ and taking the orientational average of the result, an integration by parts and some algebra yield the equation 

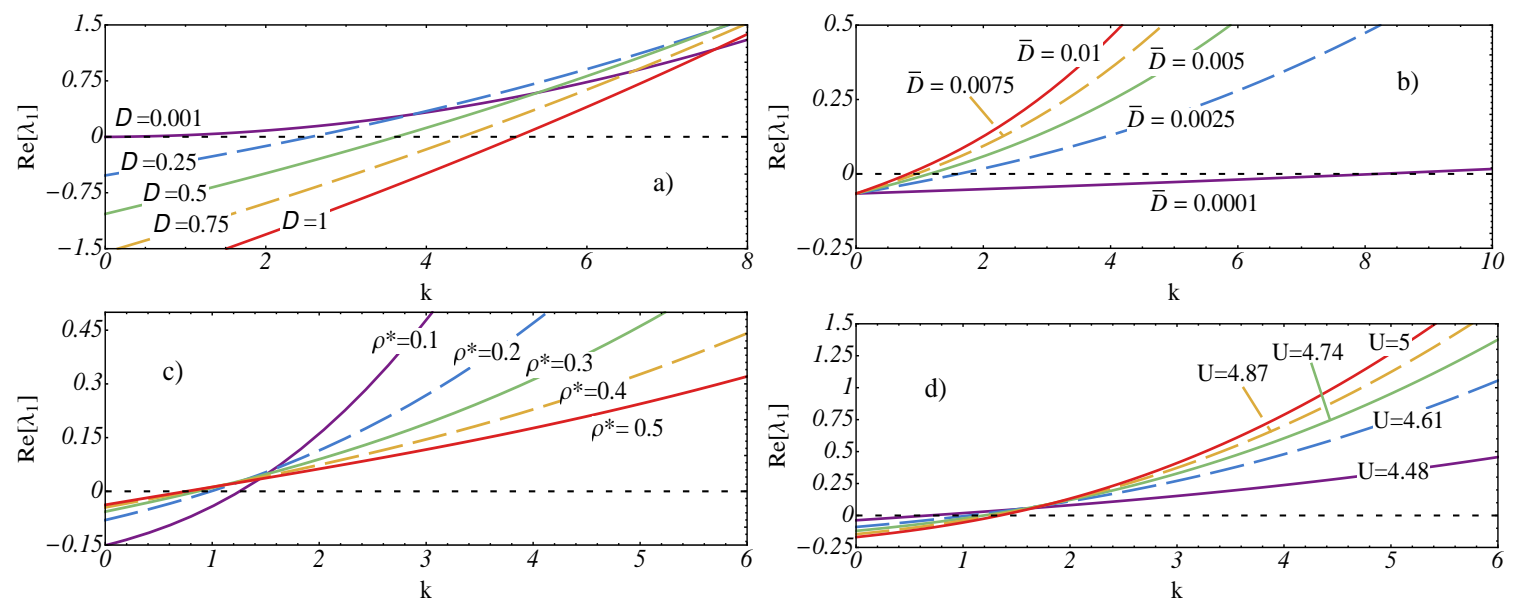

FIG. 4: The eigenvalue $\lambda_{1}$ of the linearized Eqs. (45) and (47) as a function of the wave number $k$ in $\mathrm{cm}^{-1}$ for different values of the parameters $\bar{D}$ in $\mathrm{cm}^{2} \mathrm{~s}^{-1}$ (top); $\mathcal{D}$ in $s^{-1}$ and $\rho^{*}$ in $\mathrm{cm}^{-3}$ (bottom). The eigenvalue becomes negative for $\rho^{*}$ larger than a certain critical value $\rho^{*}>\rho_{c}$. This means that large densities prevent the transition and the appearance of patterns.

$$
\begin{aligned}
\frac{\partial S_{i j}}{\partial t}= & -6 \mathcal{D} S_{i j}-2 \frac{\mathcal{D}}{k_{B} T}\left\langle\left[\vec{u} \frac{\partial \mathcal{U}}{\partial \vec{u}}\right]_{i j}^{s}-u_{i} u_{j} u_{k} \frac{\partial \mathcal{U}}{\partial u_{k}}\right\rangle \\
& +\bar{D} \nabla^{2} S_{i j}+D_{a} \nabla_{k} \nabla_{j}\left\langle\left(u_{i} u_{j}-\frac{1}{3} \delta_{i j}\right)\left(u_{k} u_{l}-\frac{1}{3} \delta_{k l}\right)\right\rangle \\
& +\frac{\bar{D}}{k_{B} T} \nabla_{k}\left\langle\left(u_{i} u_{j}-\frac{1}{3} \delta_{i j}\right) \nabla_{k} \mathcal{U}\right\rangle \\
& +\frac{D_{a}}{k_{B} T} \nabla_{k}\left\langle\left(u_{i} u_{j}-\frac{1}{3} \delta_{i j}\right)\left(u_{k} u_{l}-\frac{1}{3} \delta_{k l}\right) \nabla_{l} \mathcal{U}\right\rangle,
\end{aligned}
$$

where the symbol $\partial / \partial \vec{u}$, represents the gradient operator in $\vec{u}$-space. It is worth stressing that in the proper limiting situations, Eq. (46) reduces to diverse dynamic equations appearing in

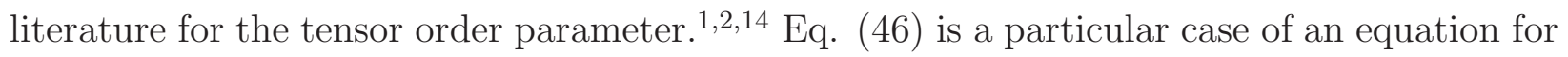
polydomain nematic liquid crystals under shear stresses used to derive microscopic formulae for the Frank-Ericksen elastic coefficients. $\frac{16}{}$ As indicated previously, Eqs. (45) and (46) constitute a set of two coupled reaction-diffusion type partial differential equations for the 

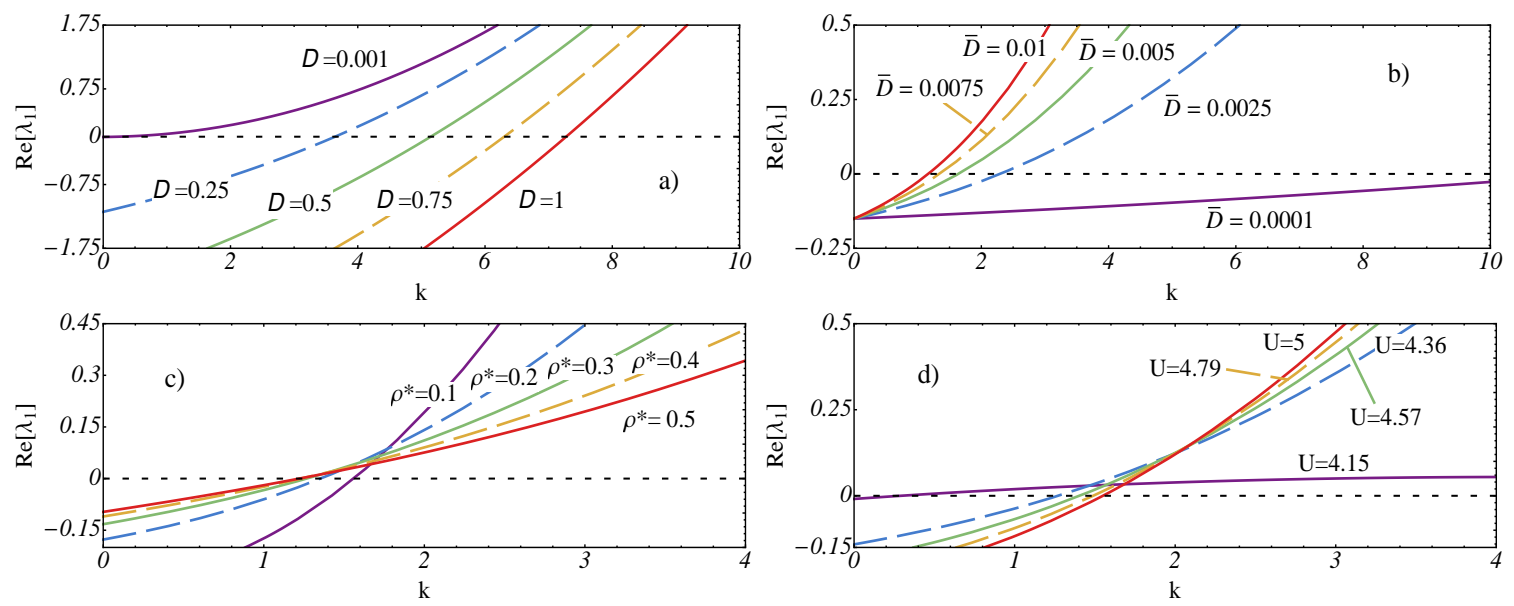

FIG. 5: The eigenvalue $\lambda_{1}$ of the linearized Eqs. (45) and (48) as a function of the wave number $k$ in $\mathrm{cm}^{-1}$ for different values of the parameters $\bar{D}$ in $\mathrm{cm}^{2} \mathrm{~s}^{-1}$ (top); $\mathcal{D}$ in $s^{-1}$ and $\rho^{*}$ in $\mathrm{cm}^{-3}$ (bottom). As in the previous case, the eigenvalue may become negative for $\rho^{*}$ larger than a certain critical value $\rho^{*}>\rho_{c}$.

scalar fields $U(\vec{r}, t)$ and $S(\vec{r}, t)$.

With the aim to simplify the description, let us assume that $D_{a}=0$ and consider that the effective diffusion coefficient of the order parameter, $\bar{D}$, is again a scalar constant quantity. As a consequence of these assumptions and using Eqs. (32) and (36) the evolution equation for $S(\vec{r}, t)$ reduces to

$$
\frac{\partial S}{\partial t}=\bar{D} \nabla^{2} S-6 \mathcal{D}\left[\left(1-\frac{U}{5}\right) S-\frac{1}{7} U S^{2}+\frac{12}{35} \nu U S^{3}+\frac{6}{35} \nu(1-\nu) U S^{4}\right]
$$

for the five order expansion of the free-energy like function in the parametric KAC closure, $\frac{18}{}$ and

$$
\frac{\partial S}{\partial t}=\bar{D} \nabla^{2} S-6 \mathcal{D}\left[\left(1-\frac{U}{5}\right) S-\frac{U}{7} S^{2}+\frac{12}{35} U S^{\frac{13}{3}}\right]
$$

for the dynamic closure. 

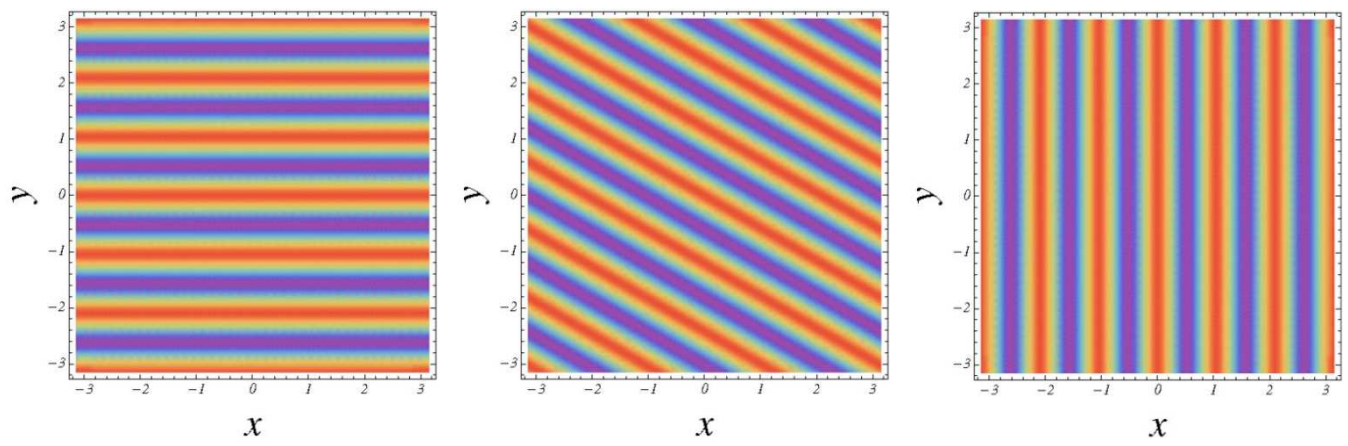

FIG. 6: Spatial structures of $\delta S$ for different components of a wave vector with magnitude $k=$ $6 \mathrm{~cm}^{-1}$. The colored regions correspond to different values of $S$ between 0 and 1 . The dark purple regions correspond to the isotropic state $(S=0)$ whereas the red regions correspond to a state with higher nematic order $(S=0.63)$. The lighter regions separating the previous ones correspond to a nematic order between 0 and 0.63 .

The existence of stationary patterns can be proved by analyzing Lyapunov's stability around the nematic equilibrium state $S_{0}$ shown by the free-energy like functions (32) and (36). In order to do this, we have to linearize the system of coupled equations (45) and (47) (for the parametric closure), and (45) and (48) (for the dynamic closure) about $S_{0}$ by assuming $S=S_{0}+\delta S$ and $U=U_{0}+\delta U \stackrel{37}{\underline{n}}$ This procedure allows us to calculate the elements of the matrix $\underline{\underline{\Lambda}}$ defining the resulting linear transformation $\underline{\dot{X}}=\underline{\underline{\Lambda}} \cdot \underline{X}$, with $\underline{X}=(\delta S, \delta U)$ and $\underline{X}$ the corresponding time derivative. The corresponding elements of these matrices are given in the Appendix A.

The solution of the perturbative system $\underline{\dot{X}}=\underline{\underline{\Lambda}} \cdot \underline{X}$ can be proposed in terms of the 
combination

$$
\underline{X}(\vec{r}, t)=\sum_{\alpha} c_{\alpha} \underline{\Psi}_{\alpha} e^{i \vec{k} \cdot \vec{r}+\lambda_{k} t}
$$

where $\lambda_{\alpha}$ and $\underline{\Psi}_{\alpha}$ are the corresponding eigenvalues and eigenvectors of $\underline{\underline{\Lambda}}$.

By performing a numerical study, it can be shown that both eigenvalues are complex. In both cases one of the eigenvalues has a negative real part for all the combinations of values of the parameters $\bar{D}, \rho^{*}$ and $U_{0}$, whereas the other one may have a positive real part for certain combinations of the parameters. Figures 4 and 5 show the corresponding results for the parametric and dynamic closures, respectively. These results imply that for the adequate combinations of parameters, i.e. physical conditions, the system presents patterns

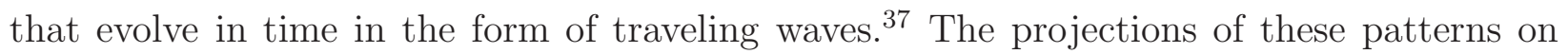
the $x-y$ plane are illustrated in Figure 6 for a wave vector of magnitude $k=6 \mathrm{~cm}^{-1}$ and three different combinations of its components. Figure 7 illustrates the propagation of the patterns in time, for the same wave number.

Finally, we have also analyzed the case of the exact IHK equilibrium closure given in Ref. 19 by assuming that the spatial and temporal evolution of the scalar order parameter may be determined by an equation similar to Eqs. (45) and (47), and according to the phenomenological approach. The existence of patterns and traveling waves is also possible in that case.

\section{DISCUSSION AND CONCLUSIONS}

In this article, we calculated a Fokker-Planck equation for the single-particle nonhomogeneous distribution function of particle orientations by assuming an interaction energy 

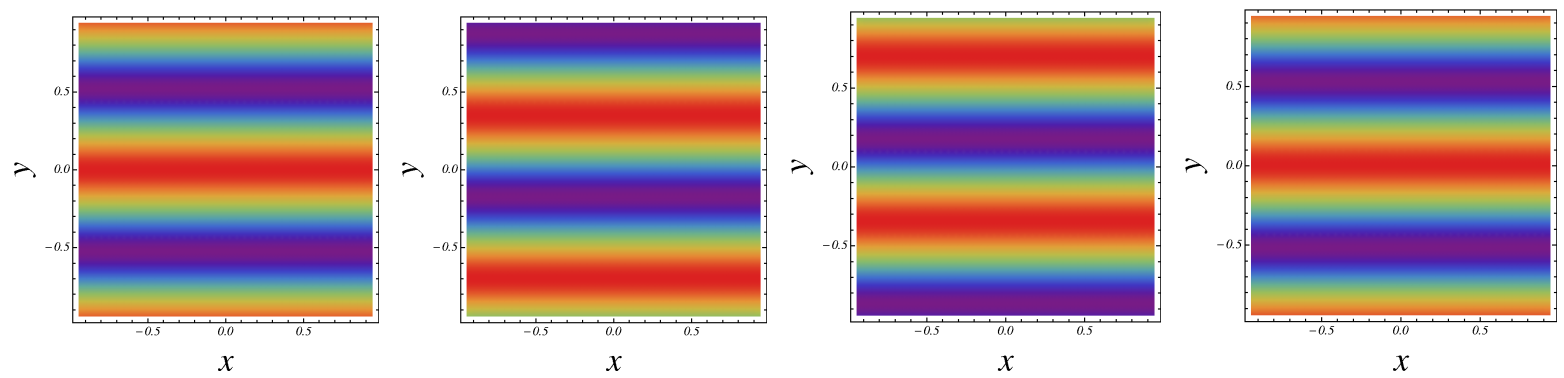

FIG. 7: $\delta S$ as a propagating wave with $k=6 \mathrm{~cm}^{-1}$. Each panel shows the configuration of the system for $t=0,4,8,12 s$. Colors mean the same as in Fig. 5 .

of the Maier-Saupe type, which couples the mesoscopic degrees of freedom with the average value of the tensor order parameter $S_{i j}$. The FPE was derived by using the Gibbs entropy postulate (3), that is also used for equilibrium situations, and calculating the entropy production of the system during relaxation. The obtained FPE is consistent with previous descriptions of the dynamics of liquid crystalline phases. $\underline{\underline{1}} \underline{\underline{2}} \underline{\underline{16}}$

Afterwards, we used the FPE to derive the first two coupled evolution equations for the moments of the distribution, Eqs. (17) and (18) and truncated the corresponding hierarchy by adopting two dynamic closure approaches in which the expression of the scalar fourth order parameter is expressed in terms of the scalar second order one. The first parametric closure, is equivalent to the exact closure reported in Ref $\underline{18}$ whereas the second closure, called dynamic, represents a novel closure that takes into account the leading term in the relaxation dynamics of the second and fourth scalar order parameters, Eqs. (25) and (26). This novel closure has a good performance when compared with the parametric closure of Ref. $\frac{18}{}$ and with the exact equilibrium closure of Ref. $\stackrel{19}{ }$, as shown in Figures 1 and 2, and Table 
1. A comparative discussion on the performance of the parametric and dynamic closures in the presence of shear flow was also done. From it may conclude that the dynamic closure relation, Eq. (34), performs quantitatively very well when compared with the parametric closure Eq. (28), and consequently can be also used to approximate the exact solution with high precision at least for the range of values considered here.

The non-equilibrium part of our analysis was motivated by the fact that Eqs. (25) and (26) are coupled in a similar way to Lotka-Volterra equations and, consequently, they allow for the possibility of oscillating behaviors. 18 We search for these behaviors in Section 5 , where we derived two coupled partial differential equations of the diffusion-reaction type for the scalar second order parameter $S$ and the non-homogeneous degree of coupling $U$. A linear stability analysis of these coupled evolution equations showed that patterns and traveling waves are indeed possible non-equilibrium solutions for the closure approximations discussed in this paper and even for the case of the equilibrium closure of Ref. [19].

In summary, we studied the dynamics of uniaxial nematic systems by using the mesoscopic non-equilibrium thermodynamics formalism in the context of a mean-field theory and within the approach of dynamic closures for the evolution equations for the orientational order parameters. The results emerging from this analysis allows us to state that the nonequilibrium structures associated to pattern formation and traveling waves are possible for these systems without the influence of an external driving. 


\section{Appendix A: Explicit form of the linearized equations for $\delta S$ and $\delta U$}

As mentioned in Sec.V, the linearization of the coupled equations for $U$ and $S$ about $S_{0}$ by assuming $S=S_{0}+\delta S$ and $U=U_{0}+\delta U$ allows us to calculate the elements of the matrix

$\underline{\underline{\Lambda}}$ that enters in the linear transformation $\underline{\dot{X}}=\underline{\underline{\Lambda}} \cdot \underline{X}$. The elements of the corresponding Jacobian associated to the parametric closure, Eqs. (45) and (47) are

$$
\begin{array}{r}
\Lambda_{s s}=\bar{D} \nabla^{2}-\frac{6}{35} \mathcal{D}\left[\left(24 S_{0}^{3} U_{0} \nu^{2}-24 S_{0}^{3} U_{0} \nu-36 S_{0}^{2} U_{0} \nu+10 S_{0} U_{0}+7 U_{0}-35\right)\right] \\
\Lambda_{s \rho}=-\frac{6}{35} \mathcal{D}\left[S_{0}\left(6 S_{0}^{3} \nu^{2}-6 S_{0}^{3} \nu-12 S_{0}^{2} \nu+5 S_{0}+7\right)\right], \\
\Lambda_{\rho \rho}=-\frac{5 \bar{D}}{\rho^{*}}\left[3 S_{0} \nabla U_{0} \cdot \nabla+3 \nabla S_{0} \cdot \nabla U_{0}+2 S_{0} \nabla^{2} U_{0}+2 U_{0} \nabla S_{0} \cdot \nabla+U_{0} S_{0} \nabla^{2}+U_{0} \nabla^{2} S_{0}\right] \\
\Lambda_{\rho s}=\bar{D} \nabla^{2}-\frac{5 \bar{D}}{\rho^{*}}\left[3 S_{0} \nabla S_{0} \cdot \nabla+S_{0}^{2} \nabla^{2}+\nabla S_{0} \cdot \nabla S_{0}+S_{0} \nabla^{2} S_{0}\right] .
\end{array}
$$

In order to search for patterns, we have to propose solutions of the form $\underline{X}=\underline{X}(t) e^{i \vec{k} \cdot \vec{r}}$ with $\vec{k}$ the wave vector of the perturbation. Assuming that in Eqs. (A1)-(A4) we may approximate $\nabla S_{0} \sim S_{0} / \mathcal{L}$ and $\nabla S_{0} \cdot \nabla \sim\left(S_{0} / \mathcal{L}\right) i k$, we finally obtain the Fourier transformed relations

$$
\begin{array}{r}
\Lambda_{s s}=-k^{2} \bar{D}-\frac{6}{35} \mathcal{D}\left[\left(24 S_{0}^{3} U_{0} \nu^{2}-24 S_{0}^{3} U_{0} \nu-36 S_{0}^{2} U_{0} \nu+10 S_{0} U_{0}+7 U_{0}-35\right)\right] \\
\Lambda_{s \rho}=-\frac{6}{35} \mathcal{D}\left[S_{0}\left(6 S_{0}^{3} \nu^{2}-6 S_{0}^{3} \nu-12 S_{0}^{2} \nu+5 S_{0}+7\right)\right] \\
\Lambda_{\rho \rho}=-\frac{5 \bar{D}}{\rho^{*}} S_{0} U_{0}\left[5 \frac{i k}{\mathcal{L}}+\frac{6}{\mathcal{L}^{2}}-k^{2}\right] \\
\Lambda_{\rho s}=-k^{2} \bar{D}-\frac{5 \bar{D}}{\rho^{*}} S_{0}^{2}\left[3 \frac{i k}{\mathcal{L}}+\frac{2}{\mathcal{L}^{2}}-k^{2}\right]
\end{array}
$$

where $\mathcal{L}=[\bar{D} / \mathcal{D}]^{1 / 2}$ is a characteristic length of the system.

In similar form, the elements of the corresponding Jacobian associated to the dynamic 
closure, Eqs. (45) and (48) are

$$
\begin{array}{r}
\Lambda_{s s}=\bar{D} \nabla^{2}-\frac{6}{35} \mathcal{D}\left[\left(52 S_{0}^{10 / 3}-10 S_{0}-7\right) U_{0}+35\right], \\
\Lambda_{s \rho}=-\frac{6}{35} \mathcal{D}\left[S_{0}\left(12 S_{0}^{10 / 3}-5 S_{0}-7\right)\right], \\
\Lambda_{\rho \rho}=-\frac{5 \bar{D}}{\rho^{*}}\left[3 S_{0} \nabla U_{0} \cdot \nabla+3 \nabla S_{0} \cdot \nabla U_{0}+2 S_{0} \nabla^{2} U_{0}+2 U_{0} \nabla S_{0} \cdot \nabla+U_{0} S_{0} \nabla^{2}+U_{0} \nabla^{2} S_{0}\right], \\
\Lambda_{\rho s}=\bar{D} \nabla^{2}-\frac{5 \bar{D}}{\rho^{*}}\left[3 S_{0} \nabla S_{0} \cdot \nabla+S_{0}^{2} \nabla^{2}+\nabla S_{0} \cdot \nabla S_{0}+S_{0} \nabla^{2} S_{0}\right] .
\end{array}
$$

The corresponding Fourier transformed relations are in this case

$$
\begin{array}{r}
\Lambda_{s s}=-k^{2} \bar{D}-\frac{6}{35} \mathcal{D}\left[\left(52 S_{0}^{10 / 3}-10 S_{0}-7\right) U_{0}+35\right], \\
\Lambda_{s \rho}=-\frac{6}{35} \mathcal{D}\left[S_{0}\left(12 S_{0}^{10 / 3}-5 S_{0}-7\right)\right], \\
\Lambda_{\rho \rho}=-\frac{5 \bar{D}}{\rho^{*}} S_{0} U_{0}\left[5 \frac{i k}{\mathcal{L}}+\frac{6}{\mathcal{L}^{2}}-k^{2}\right], \\
\Lambda_{\rho s}=-k^{2} \bar{D}-\frac{5 \bar{D}}{\rho^{*}} S_{0}^{2}\left[3 \frac{i k}{\mathcal{L}}+\frac{2}{\mathcal{L}^{2}}-k^{2}\right] .
\end{array}
$$

\section{Acknowledgments}

We acknowledge Prof. M. Rubi by critically reading this manuscript. HH acknowledges the postdoctoral program by UNAM-DGAPA for financial support. ISH and DMH thank UNAM-DGAPA for partial financial support of Grants No. IN102609 and ID100112-2.

1 M. Kröger, Models for polymeric and anisotropic fluids, vol. 675 of Lecture Notes in Physics (Springer, Berlin, 2005).

2 M. Doi and S. F. Edwards, The Theory of Polymer Dynamics, (Oxford University, New York, 1998). 
3 P. G. de Gennes and J. Prost, The Physics of Liquid Crystals, Clarendon, Oxford (1993).

4 L. Onsager, Ann. N.Y. Acad. Sci. 51, 627 (1949).

5 W. Maier and A. Saupe, Z. Naturforsch., 14a (1959) 882;

W. Maier and A. Saupe, Z. Naturforsch., 15a (1960) 287.

6 P. G. de Gennes, Phys. Lett., 30A, (1969) 454; P. G. de Gennes, Mol. Crys. Liq. Crys., 12, (1971) 193.

7 S. Hess, Z. Naturforsch. A 31, (1976) 1034.

8 E.J. Hinch and L.G. Leal, J. Fluid Mech. 76, 187 (1976).

9 D. Thirumalai, Phys. Rev. E 84, 5869 (1986).

10 R. G. Larson, Macromolecules 23, 3983 (1990).

11 H. Ehrentraut, S. Hess, Phys. Rev. E 51, 2203 (1995).

12 Q. Wang, J. Non-Newtonian Fluid Mech. 72, 142 (1997).

13 C. V. Chaubal and L. G. Leal, J. Rheol. 42, 177 (1998).

14 P. Ilg, I. V. Karlin, and H. C. Öttinger, Phys. Rev. E 60, 5783 (1999).

15 E. Hershkovits, R. Hernández, J. Chem. Phys. 122, 014509 (2005).

16 M. Kröger and P. Ilg, J. Chem. Phys. 127034903 (2007).

17 O. Alarcón-Waess, J. Chem. Phys. 132, 144902 (2010).

18 M. Kröger, A. Ammar, and F. Chinesta, J. Non-Newtonian Fluid Mech. 149, (2008) 40.

19 P. Ilg, M. Hutter, M. Kröger, Phys. Rev. E 83, 061713 (2011).

20 C. Bingham, Ann. Stat. 2, 1201 (1974).

21 W. Muschik, B. Su, J. Chem. Phys. 107, 580 (1997). 
D. Reguera, J. M. Rubi, J. M. G. Vilar, J. Phys. Chem. B 109 (2005) 21502-21515.

J. M. Rubi, A. Pérez-Madrid, Physica A 298, 177 (2001).

24 J. G. Méndez-Bermudez and I. Santamaría-Holek, Physica A 389, 1819-1828 2010.

25 H. Híjar, J. G. Méndez-Bermúdez and I. Santamaría-Holek, J. Chem. Phys. 132, 0845022010.

26 Z. Konstantinovic, M. García del Muro, M. Kovylina, X. Batlle, A. Labarta, Phys. Rev. B 79, 094201 (2009).

27 I. Santamaría-Holek, A. Pérez-Madrid, J. M. Rubi, J. Chem. Phys. 120, 2818 (2004).

28 R. Kubo, M. Toda and N. Hashitsume, Statistical Physics II. Nonequilibrium Statistical Mechanics, Second Edition, (Springer, Berlin, 1991)

29 I. Santamaría-Holek, D. Reguera, J. M. Rubi, Phys. Rev. E 63, 051106 (2001).

30 I. Santamaría-Holek, G. Barrios, J. M. Rubi, Phys. Rev. E 79, 031201 (2009).

31 T. L. Hill, An Introduction to Statistical Thermodynamics, (Dover, New York, 1986).

32 R. G. Larson, H. C. Öttinger, Macromolecules 24, 6270 (1991).

33 S. Hess, D. Frenkel, M. P. Allen, Mol. Phys. 74, 765 (1991).

34 R. G. Larson, D. W. Mead, Liq. Cryst. 12, 751 (1992).

35 M. G. Forest, Q. Wang, Hong Zhou, J. Rheol. 43, 1573 (1999).

36 M. G. Forest, Q. Wang, Hong Zhou, J. Rheol. 48, 175 (2004).

37 D. Kondepudi, I. Prigogine, Modern thermodynamics, (John Wiley \& Sons., New York, 1999). 